\title{
ORIGINAL
}

\section{ELABORACIÓN DE CRITERIOS E INDICADORES PARA DESARROLLAR Y EVALUAR PROGRAMAS DE ENVEJECIMIENTO SALUDABLE EN LOS LUGARES DE TRABAJO $(*)$}

\author{
Inmaculada Mateo Rodríguez $(1,2,3)$, Antonio Daponte Codina $(1,2,3,4)$, Mariola Bernal \\ Solano (2) y Ma José Sánchez Pérez $(1,2,3)$.
}

(1) Escuela Andaluza de Salud Pública. Granada.

(2) Centro de Investigación Biomédica en Red de Epidemiología y Salud Pública (CIBERESP). Instituto de Salud Carlos III.

(3) Instituto de Investigación Biosanitaria IBS. Hospitales Universitarios de Granada/Universidad de Granada. Granada. España

(4) Observatorio de Salud y Medio Ambiente de Andalucía (OSMAN). Consejería de Salud de la Junta de Andalucía.

(*) Este trabajo se enmarca en el Proyecto PROGRESS: Toward healthy ageing in Europe, financiado por el Programa comunitario para el empleo y la solidaridad social (PROGRESS) de la Unión Europea: VS/2011/0489, en el que participaron cinco países europeos.

Los autores declaran que no existe conflicto de intereses en la realización del estudio. Este trabajo forma parte de una tesis doctoral dentro del Programa de Doctorado en Ciencias de la Salud de la Universidad de Sevilla, Universidad de Jaén y la EASP.

\section{RESUMEN}

Fundamentos: En el actual contexto poblacional de envejecimiento de la población y prolongación de la vida laboral, los programas de promoción de salud en el trabajo son una herramienta clave y necesaria para promover un envejecimiento activo y saludable. El objetivo fue elaborar una herramienta tipo lista de verificación para orientar la planificación, implementación y evaluación de buenas prácticas en intervenciones en este ámbito, aplicables a una variedad de programas, países y centros de trabajo.

Métodos: Se utilizó una técnica Delphi en tres rondas en la que participaron expertos/as de cinco países europeos. Se elaboró un cuestionario en línea a partir de una lista de criterios e indicadores y, empleando el correo electrónico, se trabajó durante las sucesivas rondas en su adaptación a la evaluación de intervenciones. Mediante procesos de valoración y consenso, se priorizaron los criterios e indicadores más relevantes.

Resultados: Partiendo de nueve criterios basales se priorizaron cuatro criterios clave cuyas puntuaciones medias fueron: relevancia: 62, adecuación al objetivo: 57 , innovación: 50 y garantía de calidad: 41 . Utilizando esta matriz de criterios e indicadores se diseñó una lista de verificación con la información clave que debe recogerse a la hora de planificar, implementar y evaluar buenas prácticas en las intervenciones.

Conclusiones: La lista de verificación ayuda a sistematizar la metodología global de implantación de intervenciones que puede ser utilizada por responsables de los programas para promover el envejecimiento activo y saludable en los centros de trabajo.

Palabras clave: Salud laboral. Envejecimiento. Planificación en salud. Correspondencia

Inmaculada Mateo Rodríguez

Escuela Andaluza de Salud Pública

Campus Universitario de Cartuja

Cuesta del Observatorio, 4

Apartado de correos 2070

18080 Granada

inmaculada.mateo.easp@juntadeandalucia.es

DOI:

\section{ABSTRACT \\ Elaboration of Criteria and Indicators to Develop and Evaluate Programs of Healthy Aging in the Workplace}

Background: In the actual context of population ageing and extension of working age, programs for health promotion at the workplace are a key and necessary tool to promote an active and healthy ageing. This work presents the methodological process followed to elaborate a checklist tool, within the framework of the European project Progress, that contributes to orientate planning, implementation and evaluation of good practices in this field, to be applicable to a variety of programs, countries and workplaces.

Methods: A Delphi technique has been applied in three rounds in which experts in the area from five European countries participated. A questionnaire created from a list of criteria and indicators was adapted throughout the rounds, with the use of webmail, to the evaluation of interventions in the field of interest. Through processes of assessment and consensus, criteria and indicators most relevant were prioritized.

Results: From the nine starting criteria and after the implementation of the technique, four key criteria were prioritized: relevance: 62 , adequacy to objective: 57 , innovation: 50 and guarantee of quality: 41 . Using this group of criteria and indicators, a checklist was designed containing the key information that should be collected for planning, implementation and evaluation of good practices in interventions in this field.

Conclusions: The checklist tool helps to systematize the global methodology for the implementation of interventions which could be very useful for persons responsible of programmes to promote active and healthy ageing in the workplace.

Key words: Occupational health. Ageing. Guidelines for health planning.

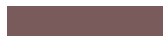




\section{INTRODUCCIÓN}

El aumento de la esperanza de vida de la población representa uno de los mayores triunfos de la humanidad, resultado del éxito de las políticas de salud pública y bienestar y del desarrollo social y económico, al tiempo que es uno de los principales desafíos estratégicos de nuestro país y los países de nuestro entorno europeo para los próximos años ${ }^{1}$. Según los últimos datos publicados por Eurostat $(2015)^{2}$, la población mayor de 65 años en la Unión Europea (UE-28) pasará del 18,2\% en 2013 al $28,7 \%$ en 2080 . España es uno de los países principalmente afectados por el envejecimiento, con un índice de envejecimiento (cociente entre la población mayor de 64 años y la menor de 16 años, expresado en porcentaje) que ha pasado del $35,9 \%$ en el año 1975 al $114,7 \%$ en 2015 . Se estima que a partir de 2017 el número de defunciones superará por primera vez al de nacimientos $^{3}$. Es por ello que el desarrollo de medidas para la promoción de un envejecimiento activo y saludable (EAS) es un objetivo político de primer orden en Europa ${ }^{4}$. La Organización Mundial de la Salud define el envejecimiento activo como "el proceso en el que se optimizan las oportunidades de salud, participación y seguridad a fin de mejorar la calidad de vida de las personas a medida que envejecen"". Esta manera de conceptualizar el envejecimiento, como un proceso que ocurre a lo largo de la vida, implica poner el eje de las políticas no solo en las acciones dirigidas a poblaciones de más de 65 años sino en influir sobre los factores que operan a lo largo de toda la vida y que determinan la salud y autonomía en las edades avanzadas. Estos factores tienen que ver en gran parte con las condiciones en las que se vive y se trabaja a lo largo de todo el ciclo vital ${ }^{5}$.

Los cambios demográficos están influyendo en la esfera laboral de manera que el porcentaje de trabajadores de 55 a 64 años en el mercado de trabajo (de 20 a 64 años) se estima que crecerá en torno a un tercio, aumentando del $13,7 \%$ en 2013 a $18,3 \%$ en 2060 en el conjunto de la UE. Sin embargo en varios países, entre los que se encuentra España, este aumento se situará por encima del $50 \%{ }^{6}$.

En respuesta al reto que supone el envejecimiento de la población para la sostenibilidad de los sistemas de bienestar se están desarrollando iniciativas sociales y políticas orientadas a prolongar la vida laboral. Es el caso en España con la Ley de adecuación, adaptación y modernización del sistema de Seguridad Social ${ }^{7}$ que amplía la edad de jubilación hasta los 67 años, así como de la Estrategia Global para el Empleo de los Trabajadores y las Trabajadoras de Más Edad 2012-2014 (Estrategia 55 y más $)^{8}$, ambas reflejo de estas directrices. Estas propuestas se enfrentan sin embargo con un desafío importante, ya que el incremento en la esperanza de vida en los países europeos no ha implicado un aumento similar en la esperanza de vida libre de discapacidad (EVLD). Según un informe de la Comisión sobre Determinantes Sociales de la Salud de la OMS $(2010)^{9}$ en los próximos años la mitad de la población del Reino Unido tendrá una discapacidad a los 62 años y la EVLD será aún menor para los grupos sociales y laborales más desfavorecidos. El estudio también sugiere que el empeoramiento en el funcionamiento físico se da 12 años antes en trabajadores/as manuales que en quienes tienen ocupaciones profesionales y de mayor estatus social. En España la EVLD al nacer es de 64,7 años para los hombres y 63,9 para las mujeres ${ }^{10}$.

Los resultados de la Encuesta de Salud, Envejecimiento y Jubilación en Europa ${ }^{11}$ evidencia la importancia del estado de salud de las personas a la hora de analizar su participación en el mercado laboral y señala que el empeoramiento del estado de salud asociado a la edad explica en gran medida la menor participación laboral a partir de los 50 años. El 40\% de trabajado- 
ras/es en Europa declara que no será capaz de seguir desempeñando su trabajo actual a los 60 años. En España, los datos de la Encuesta Nacional de Salud 2012 muestran que la prevalencia de enfermedades crónicas aumenta con la edad tanto en hombres como en mujeres ${ }^{12}$.

Ante este escenario, se hace especialmente urgente que las políticas dirigidas a la prolongación de la vida laboral vayan acompañadas de estrategias que generen condiciones de trabajo seguras y saludables ante una diversidad de personas con diferentes edades y condiciones de salud. Los programas de promoción de salud en los lugares de trabajo (PSLT) constituyen una herramienta clave para avanzar en esa dirección al ofrecer un marco de acción e instrumentos concretos para promover entornos laborales que generen oportunidades de elecciones positivas para la salud, facilitando así que se "acumulen activos para la salud" a lo largo de la vida ${ }^{13,14}$. Estos programas son importantes para promover el EAS porque vienen produciendo conocimiento científico e instrumentos concretos para identificar precozmente situaciones de vulnerabilidad en trabajadora/es mayores, planificar intervenciones que prevengan la discapacidad o problemas crónicos de salud, favorecer entornos más inclusivos en esas circunstancias y mejorar la empleabilidad de los y las trabajadoras con enfermedad crónica ${ }^{14}$.

A pesar de la evidencia que apoya el impacto positivo de las intervenciones de promoción de salud en los lugares de trabajo $^{15,16}$, la aplicación de este tipo de intervenciones aún es escasa, sobre todo en España y otros países del sur europeo. Además, suelen presentar dos tipos de limitaciones: por lo general consisten en actuaciones puntuales más que en programas sistemáticamente planificados y basados en un modelo teórico apoyado en la evidencia científica y en pocas ocasiones se realiza evaluación, en especial del proceso, con lo que resulta difícil identificar los aspectos que deberían ser replicados en futuras intervenciones para maximizar su eficacia ${ }^{17,18}$. En este contexto se hace necesario un instrumento que ayude a orientar el diseño y la evaluación de actuaciones de PSLT de manera sistemática y planificada y que incorpore objetivos específicos para la promoción del EAS. Es este el objetivo del presente estudio.

El trabajo aquí presentado deriva del proyecto europeo Progress Towards Healthy Ageing in Europe (PROGRESS) ${ }^{19}$, que consistió en elaborar herramientas conceptuales y recursos prácticos dirigidos a la implementación de intervenciones de calidad de PSLT dirigidas al EAS, en personas trabajadoras de 45 a 68 años en centros de trabajo de diferentes países europeos.

El objetivo de la investigación fue construir una lista de verificación que defina los distintos componentes a considerar en la puesta en marcha de programas de PSLT dirigidos al EAS que sean aplicables a una variedad de países y centros de trabajo.

\section{MATERIAL Y MÉTODO}

Para construir los ítems principales de la lista de verificación para la planificación, implantación y evaluación de intervenciones en el lugar de trabajo para la promoción del EAS se decidió definir una matriz de criterios e indicadores que deberían cumplir este tipo de intervenciones. Para ello se aplicó una técnica Delphi en tres rondas durante el periodo comprendido entre abril y junio de 2013. El Delphi es un método de toma de decisiones informadas basadas en el conocimiento de personas expertas ${ }^{20}$ que recopila opiniones y crea consenso a través de un proceso iterativo estructurado mediante rondas de respuestas a un cuestionario. En cada ronda las personas participantes revisan los comentarios y evaluaciones proporcionadas por el resto del grupo en las rondas anteriores y generan una nueva respuesta sobre cada cuestión planteada. Es además un método especialmente útil 
para recoger la opinión de personas claves a distancia ${ }^{21}$.

Las personas invitadas a participar en el estudio fueron contactadas a partir de los equipos de trabajo de los cinco países implicados en el proyecto PROGRESS: Alemania, Dinamarca, España, Italia y Reino Unido. Se generó una lista de once participantes potenciales de los cuales ocho participaron en la primera ronda y nueve en la segunda y tercera ronda. Los perfiles profesionales de los componentes fueron un gestor/a de estrategias en salud, dos expertos/as en políticas de salud pública, dos gestores/as de proyectos, un consultor/a en proyectos internacionales, y tres científico/ as y académico/as del ámbito de la salud pública y la salud laboral.

Figura 1

Fases de la técnica Delphi

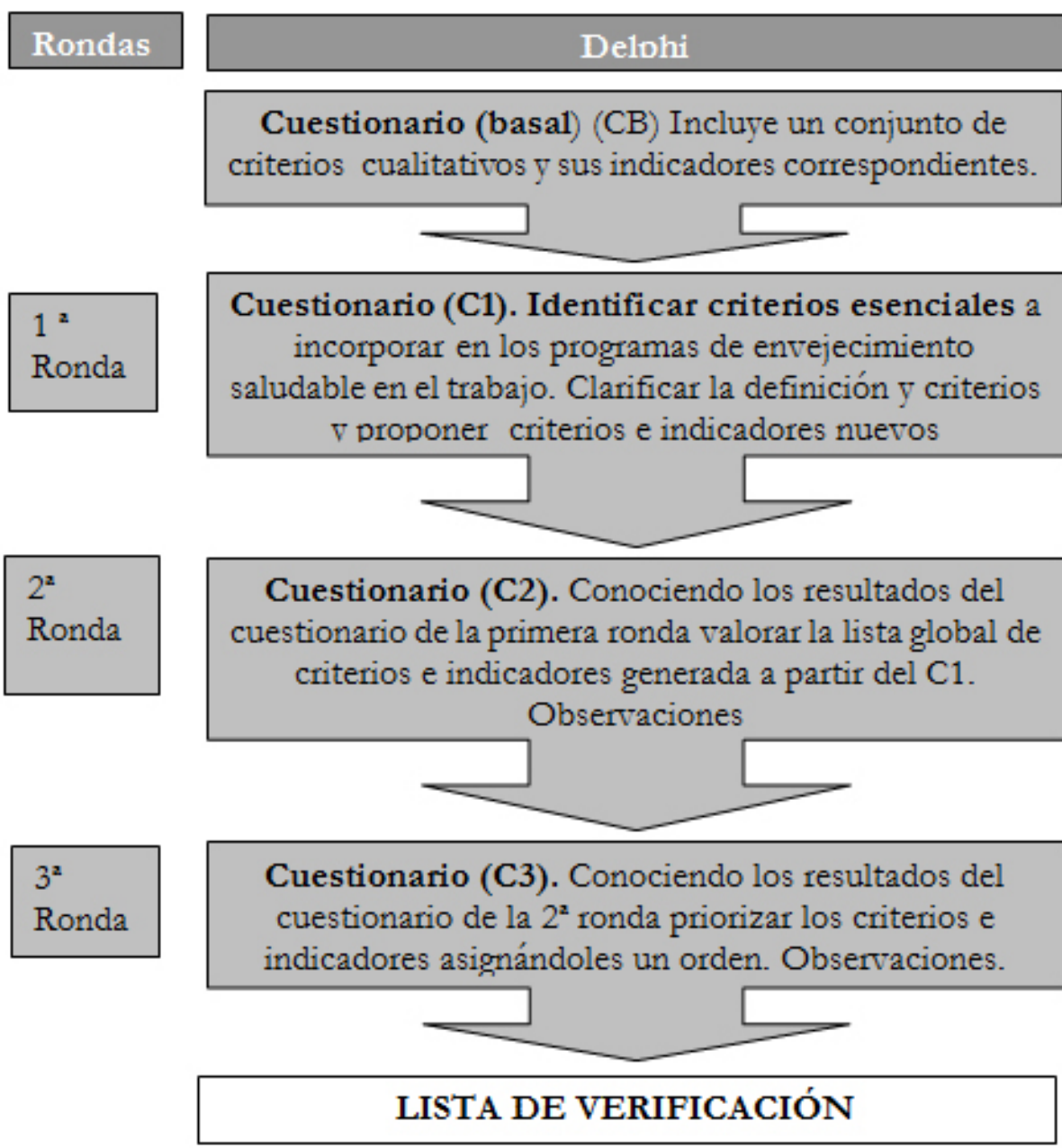


La técnica fue aplicada a través de correo electrónico siguiendo un proceso en varias etapas que se detallan en la figura 1. Se elaboraron tres cuestionarios, uno para cada ronda de recogida de información (disponibles en http://campus.easp. es/Abierto/course/view.php?id=485 previa solicitud de acceso a la primera autora del artículo).

El cuestionario basal (C1) incluía una lista de criterios generales con sus correspondientes definiciones y criterios operativos (indicadores) que ya habían sido aplicados en estudios anteriores para evaluar intervenciones diferentes en salud pública ${ }^{22-25}$. Este primer cuestionario $(\mathrm{C} 1)$ se utilizó en la primera ronda de consulta, en la que se solicitó la exclusión y/o inclusión en la matriz de criterios e indicadores útiles (o no) para la evaluación específica de intervenciones de PSLT para la promoción del EAS en el lugar de trabajo. Además, se invitó a realizar sugerencias acerca de la definición de los criterios y su adaptación al contexto específico del tipo de intervención objetivo del estudio. Esta información se incluyó en el segundo cuestionario (C2), que se volvió a enviar en línea para la siguiente consulta. En esta segunda ronda se solicitó que se valoraran los criterios e indicadores resultantes de la primera ronda mediante una escala numérica de 1 a 5 en función de su importancia y aplicabilidad. Se analizaron las puntuaciones medias obtenidas para cada criterio e indicador. Considerando esta información se construyó un tercer cuestionario (C3) que incorporó el resultado de la segunda ronda en el que se solicitó que se priorizaran los criterios e indicadores más importantes, ordenándolos de menor a mayor según su relevancia, en una escala de 1 a 10 , teniendo en cuenta los resultados y comentarios del resto del grupo en la ronda anterior respecto a cada criterio e indicador. El análisis cuantitativo de este tercer cuestionario consistió en el cálculo de terciles. Para calcularlos se utilizó la puntuación media que obtuvo el criterio. Se pidió a cada participante que ordenara los criterios dándoles un valor de 1 a 10 . Se seleccionaron los indicadores situados en el tercer tercil. Esta es una técnica utilizada frecuentemente en estudios de salud ${ }^{26-28}$. Para calcular el porcentaje de participantes se consideró el de las personas que dieron al menos 1 punto al criterio.

En todas las rondas de cuestionarios se pidió que se incorporaran comentarios para ayudar a entender la valoración que realizaban de cada criterio, los cuales eran incluidos en los cuestionarios de la siguiente ronda. Además de los análisis cuantitativos se realizó un análisis de contenido de las sugerencias aportadas en la definición de los criterios en el $\mathrm{C} 1$, así como de los comentarios que aportaban en los C2 y C3 para justificar las valoraciones de los criterios e indicadores.

En base a los resultados de la técnica Delphi aplicada, se seleccionaron los criterios y sus correspondientes indicadores que obtuvieron un mayor consenso, definido este como haber obtenido una valoración media por encima del tercer tercil. Se construyó una lista de verificación a partir de la información resultante.

\section{RESULTADOS}

El cuestionario basal (C1) partió de un total de 9 criterios cualitativos con sus correspondientes indicadores. En la primera ronda, la relevancia, la adecuación al objetivo, la innovación, la garantía de calidad y la aplicabilidad obtuvieron el máximo consenso en su consideración de criterio esencial (votado por el $100 \%$ de participantes). Además se presentaron sugerencias sobre las definiciones de los criterios, se propuso la incorporación de criterios nuevos, y se identificaron dos (efectividad de resultados y efectividad de proceso) que podían integrarse en uno solo que fue llamado "garantía de calidad". La tabla 1 muestra los resultados de esta primera fase. 


\begin{tabular}{|c|c|c|c|c|}
\hline \multicolumn{5}{|c|}{$\begin{array}{c}\text { Tabla } 1 \\
\text { Resultados principales de las rondas } 1 \text { y } 2 \text { de la técnica Delphi }\end{array}$} \\
\hline \multirow[b]{2}{*}{$\begin{array}{l}\text { Criterios } \\
\text { propuestos } \\
\text { y sugeridos }\end{array}$} & \multirow{2}{*}{$\begin{array}{c}1^{\mathrm{a}} \text { ronda } \\
\% \\
\text { puntuado } \\
\text { como esencial }\end{array}$} & \multicolumn{3}{|r|}{$2^{\mathrm{a}}$ ronda } \\
\hline & & $\begin{array}{l}\text { Importancia } \\
\text { puntuación } \\
\text { media }\end{array}$ & $\begin{array}{c}\begin{array}{c}\text { Aplicabilidad } \\
\text { puntuación } \\
\text { media }\end{array} \\
\end{array}$ & Definicion del criterio \\
\hline Relevancia & $100 \%$ & 4,8 & 4,5 & $\begin{array}{l}\text { Una intervención es considerada "relevan- } \\
\text { te" para promover un envejecimiento activo } \\
\text { y saludable (EAS) en el trabajo cuando: se } \\
\text { encuentra en el marco de una política o es- } \\
\text { trategia general (Nacional, regional o local } \\
\text { u organizacional/empresa); los objetivos de } \\
\text { la intervención corresponden con las nece- } \\
\text { sidades y prioridades identificadas por la } \\
\text { población mayor trabajadora de ese centro } \\
\text { (Correspondencia entre objetivos y nece- } \\
\text { sidades del grupo objetivo); y está dirigida } \\
\text { a los grupos de trabajadores y trabajadoras } \\
\text { mayores más vulnerables del centro (ej. } \\
\text { aquellos/as más mayores, los y las que pre- } \\
\text { sentan enfermedades crónicas y/o discapaci- } \\
\text { dad, inmigrante, otros). }\end{array}$ \\
\hline Adecuación al objetivo & $100 \%$ & 4,3 & 3,8 & $\begin{array}{l}\text { Una intervención es considerada "adecua- } \\
\text { da" para promover un EAS en el trabajo } \\
\text { cuando: está basada en la evaluación y aná- } \\
\text { lisis de los retos principales asociados al } \\
\text { envejecimiento de la población trabajadora; } \\
\text { existe correspondencia entre medios y fines } \\
\text { de la intervención; y las actividades que se } \\
\text { proponen están basadas en la evidencia dis- } \\
\text { ponible. }\end{array}$ \\
\hline Innovación & $100 \%$ & 4,4 & 3,4 & $\begin{array}{l}\text { Una intervención es considerada "inno- } \\
\text { vadora" cuando: implica nuevas ideas y/o } \\
\text { implementa una nueva manera de llegar a } \\
\text { un resultado y/o realizar un trabajo. En el } \\
\text { ámbito de la promoción del EAS en el tra- } \\
\text { bajo supone aplicar modelos conceptuales, } \\
\text { metodológicos o tecnológicos innovadores } \\
\text { (ej. marco de activos en salud, uso de TIC, } \\
\text { y otros). }\end{array}$ \\
\hline $\begin{array}{l}\text { Garantía de calidad: } \\
\text { incluye efectividad } \\
\text { de resultados } \\
\text { y de proceso }\end{array}$ & $100 \%$ & 4,2 & 4,1 & $\begin{array}{l}\text { Una intervención incorpora este criterio } \\
\text { cuando: incluye tareas de monitorización y } \\
\text { evaluación de procesos y resultados; incor- } \\
\text { pora una predicción de acciones correctivas; } \\
\text { y la población objetivo participa en la eva- } \\
\text { luación. En el ámbito de la promoción de } \\
\text { EAS en el trabajo, además las intervencio- } \\
\text { nes de calidad utilizan una perspectiva mul- } \\
\text { tidimensional según la evidencia disponible. }\end{array}$ \\
\hline Aplicabilidad & $100 \%$ & 4,2 & 4,0 & $\begin{array}{l}\text { Una intervención es considerada "aplicable" } \\
\text { cuando cuenta con el respaldo suficiente } \\
\text { para su puesta en marcha, se han identifi- } \\
\text { cado potenciales barreras y oportunidades } \\
\text { para su desarrollo (ej. las actividades que } \\
\text { se proponen son asumibles por las personas } \\
\text { a las que se dirigen considerando posibles } \\
\text { diferencias en sus capacidades psicofísicas), } \\
\text { cuenta con un plan de acción para actuar so- } \\
\text { bre los mismos, y además existe una plani- } \\
\text { ficación temporal y realista de las acciones. }\end{array}$ \\
\hline
\end{tabular}




\begin{tabular}{|c|c|c|c|c|}
\hline \multicolumn{5}{|c|}{$\begin{array}{c}\text { Tabla } 1 \\
\text { continuación }\end{array}$} \\
\hline \multirow[b]{2}{*}{$\begin{array}{l}\text { Criterios } \\
\text { propuestos y suge- } \\
\text { ridos }\end{array}$} & \multirow{2}{*}{$\begin{array}{c}1^{\mathrm{a}} \text { ronda } \\
\% \\
\text { puntuado } \\
\text { como esencial }\end{array}$} & \multicolumn{3}{|r|}{$2^{\mathrm{a}}$ ronda } \\
\hline & & $\begin{array}{c}\text { Importancia } \\
\text { puntuación } \\
\text { media }\end{array}$ & $\begin{array}{l}\text { Aplicabilidad } \\
\text { puntuación } \\
\text { media }\end{array}$ & Definicion del criterio \\
\hline Adecuación recursos & $50 \%$ & 2,8 & 2,8 & $\begin{array}{l}\text { Una intervención cumple con el criterio de ade- } \\
\text { cuación de recursos cuando existe una valoración } \\
\text { previa a su puesta en marcha de los recursos } \\
\text { humanos, materiales y financieros que va a ne- } \\
\text { cesitar, y cuenta con recursos suficientes para su } \\
\text { puesta en marcha. }\end{array}$ \\
\hline Mainstreaming & & 3,5 & 3,1 & $\begin{array}{l}\text { Este criterio se cumple cuando las metas que per- } \\
\text { sigue la intervención en cuanto a la promoción } \\
\text { del EAS en el trabajo se incorporan en todos los } \\
\text { niveles y procesos de gestión de la organización. }\end{array}$ \\
\hline Transferibilidad & $\begin{array}{l}\text { Nuevo } \\
\text { criterio } \\
\text { aportado } \\
\text { en esta ronda }\end{array}$ & 3,5 & 3,2 & $\begin{array}{l}\text { Se considera una intervención transferible cuan- } \\
\text { do se puede desarrollar en contextos diferentes a } \\
\text { aquellos para los que se diseñó inicialmente, y se } \\
\text { puede aplicar a sectores y perfiles de trabajado- } \\
\text { res/as mayores diferentes (ej. hombres y mujeres, } \\
\text { con y sin enfermedades crónicas, distintas ocupa- } \\
\text { ciones y otros). }\end{array}$ \\
\hline Equidad/Igualdad & $\begin{array}{l}\text { Nuevo } \\
\text { criterio } \\
\text { aportado } \\
\text { en esta ronda }\end{array}$ & 4,1 & 4,4 & $\begin{array}{l}\text { Una intervención considera el criterio de igual- } \\
\text { dad cuando incorpora la perspectiva de género } \\
\text { y otros posibles ejes de desigualdad (edad, clase } \\
\text { social, etnicidad, etc.) tanto en el desarrollo como } \\
\text { en la evaluación de las actividades. }\end{array}$ \\
\hline Sostenibilidad & $\begin{array}{l}\text { Nuevo } \\
\text { criterio } \\
\text { aportado } \\
\text { en esta ronda }\end{array}$ & 3,8 & 3,3 & $\begin{array}{l}\text { Una intervención es sostenible cuando se incor- } \\
\text { poran elementos que facilitan su aplicación a } \\
\text { largo plazo, (ej. cuando puede funcionar con re- } \\
\text { cursos propios y/o con estrategias que pueden ser } \\
\text { mantenidas en el tiempo. }\end{array}$ \\
\hline Ética & $\begin{array}{l}\text { Nuevo } \\
\text { criterio } \\
\text { aportado } \\
\text { en esta ronda }\end{array}$ & 3,8 & 3,7 & $\begin{array}{l}\text { La intervención cumple este criterio cuando in- } \\
\text { corpora medidas para respetar principios éticos } \\
\text { como: confidencialidad, anonimato y seguridad } \\
\text { (es lo menos invasiva posible). }\end{array}$ \\
\hline
\end{tabular}

Además de los criterios anteriores, como resultado de esta primera ronda del cuestionario se identificó una lista de indicadores que podrían utilizarse para valorar el grado en que los programas incorporaban cada criterio. El número total de indicadores propuestos en esta fase fue de 51. Fundamentalmente se identificaron indicadores asociados a los criterios garantía de calidad ( 15 indicadores), aplicabilidad (11), relevancia (9), adecuación al objetivo (9) e innovación (6). Para el resto de criterios se identificaron entre 5 y 2 indicadores diferentes para cada uno. El resultado de esta ronda fue una matriz de los 11 criterios de la tabla 1 con sus correspondientes definiciones operativas e indicadores correspondientes.
Considerando los resultados anteriores se elaboró el segundo cuestionario (C2) que fue sometido a consulta en la segunda ronda del Delphi. La tabla 1 presenta los resultados de las respuestas a este segundo cuestionario (C2). Se detallan las puntuaciones medias recibidas por los criterios en base a su importancia y aplicabilidad, así como las definiciones operativas definitivas que resultaron del análisis de contenido de las respuestas. Esta información se presentó en el tercer cuestionario de consulta (C3) para que fueran tenidas en cuenta a la hora de seleccionar los indicadores más relevantes para valorar el cumplimiento de cada criterio. 


\section{Tabla 2}

Resultados principales de la ronda 3 de la técnica Delphi. Porcentajes terciles de participantes que seleccionaron cada criterio al menos con 1 punto $(n=9)$

\begin{tabular}{|l|c|c|c|}
\hline & $\begin{array}{c}\text { Porcentaje } \\
\text { de participantes } \\
\text { que seleccionó } \\
\text { el criterio }\end{array}$ & $\begin{array}{c}\text { Puntuación } \\
\text { media }\end{array}$ & Tercil \\
\hline Relevancia & $100 \%$ & 62 & 3 \\
\hline Adecuación al objetivo & $100 \%$ & 57 & 3 \\
\hline Innovación & $100 \%$ & 50 & 3 \\
\hline Garantía de Calidad (efectividad en el proceso y en el resultado) & $100 \%$ & 41 & 3 \\
\hline Aplicabilidad & $85 \%$ & 40 & 2 \\
\hline Transferible & $100 \%$ & 32 & 2 \\
\hline Adecuación recursos & $100 \%$ & 30 & 2 \\
\hline Equidad/Igualdad & $100 \%$ & 23 & 2 \\
\hline Sostenibilidad & $85 \%$ & 18 & 1 \\
\hline Mainstreaming & $71 \%$ & 17 & 1 \\
\hline Ética & $28 \%$ & 7 & 1 \\
\hline
\end{tabular}

En la tabla 2 se presentan los resultados del tercer y último cuestionario (C3). A partir del análisis de las puntuaciones medias obtenidas, los criterios y los indicadores fueron ordenados en terciles. Los criterios que obtuvieron puntuaciones en el tercer tercil y que fueron elegidos por al menos el $80 \%$ de los/as participantes fueron seleccionados para elaborar la lista de verificación definitiva.

Cuatro fueron los criterios que cumplieron ambas condiciones:

- Relevancia (puntuación media 62): mediante estrategias que muestren que el programa es apoyado de manera clara por los más altos niveles de responsabilidad de la organización y que centran sus objetivos en las necesidades prioritarias.

- Adecuación al objetivo (puntuación media 57): que incorporen intervenciones apropiadas a los objetivos de salud de la población diana basadas en la evidencia científica disponible.

- Innovación (puntuación 50): incluyendo medidas que demuestren un esfuerzo por conocer y aplicar nuevos paradigmas, nuevos métodos y nuevas soluciones para conseguir los objetivos de salud.

- Garantía de calidad (puntuación 41): incorporando métodos e instrumentos para evaluar su aplicabilidad y efectividad así como su abordaje multidimensional.

En la tabla 3 se presentan las puntuaciones medias obtenidas por los indicadores finalmente seleccionados de los cuatro criterios priorizados (relevancia, adecuación al objetivo, innovación y garantía de calidad).

Con el fin de facilitar la aplicación de esta matriz de criterios e indicadores a la hora de planificar, implementar y evaluar programas de PSLT dirigidos a personas de más de 45 años, se construyó la lista de verificación con las cuestiones a incluir con objeto de garantizar que se cumplan los cuatro criterios anteriores. La lista de verificación puede ser utilizada tanto en la evaluación de programas como en la orientación de su planificación y diseño (anexo 1). 


\begin{tabular}{|c|c|c|c|c|}
\hline \multicolumn{5}{|c|}{ priorizados } \\
\hline & $\begin{array}{l}\text { Número de orden } \\
\text { en el que se expusieron }\end{array}$ & $\begin{array}{l}\text { Puntuación } \\
\text { media }\end{array}$ & Tercil & $\begin{array}{l}\text { Ítem de la lista de verificación } \\
\text { para evaluar ese indicador }\end{array}$ \\
\hline \multicolumn{5}{|c|}{ RELEVANCIA } \\
\hline $\begin{array}{l}\text { Existe una política organizacional en PSLT o cualquier documento en el centro de trabajo (convenio } \\
\text { colectivo, política de Seguridad y Salud en el trabajo, u otros) donde se prioriza la promoción de } \\
\text { salud y el bienestar de los y las trabajadoras en general y de los grupos de mayor edad en particular } \\
\text { y se define el compromiso por establecer recursos para el desarrollo de intervenciones. }\end{array}$ & 1 & 58 & $3^{\circ}$ & 1,2 \\
\hline $\begin{array}{l}\text { Existe una evaluación inicial de la salud de los y las empleadas que ayuda a priorizar los aspectos } \\
\text { más relevantes sobre los que intervenir, así como a identificar áreas de mejora y necesidades no } \\
\text { cubiertas. }\end{array}$ & 2 & 56 & $3^{\circ}$ & $3,5 . a$ y $5 . b$ \\
\hline $\begin{array}{l}\text { Los objetivos del programa se definen con la participación de lo/as trabajadoras. Las prioridades } \\
\text { que se establecen cuentan con su aceptación. }\end{array}$ & 5 & 52 & $3^{\circ}$ & $5 . c$ y $5 . d$ \\
\hline $\begin{array}{l}\text { La intervención prioriza la población objetivo en función de ciertas características (ej. grupos de } \\
\text { mayores, en ocupaciones de mayor peligrosidad, o menor estatus como mujeres, población inmi- } \\
\text { grante, personas con enfermedad crónica u otras) }\end{array}$ & 9 & 40 & $3^{\circ}$ & $4,5 . \mathrm{e}$ \\
\hline $\begin{array}{l}\text { Se desarrollan actuaciones para la conciliación de la vida laboral, familiar y personal entre los } \\
\text { y las trabajadoras, considerado un factor importante para la calidad de vida en población mayor } \\
\text { trabajadora. }\end{array}$ & 6 & 33 & $2^{\circ}$ & NP \\
\hline $\begin{array}{l}\text { La dirección del centro apoya el envejecimiento saludable y lo demuestra organizando actividades y } \\
\text { el acceso a recursos para su desarrollo }\end{array}$ & 7 & 30 & $2^{\circ}$ & NP \\
\hline $\begin{array}{l}\text { Se incorporó en los registros habituales del centro información sobre aquello que puede ser impor- } \\
\text { tante para identificar necesidades en salud (como datos de absentismo, registros sanitarios, etc.) } \\
\text { hacia donde orientar los programas de EAS. }\end{array}$ & 4 & 25 & $1^{\circ}$ & NP \\
\hline $\begin{array}{l}\text { El análisis de situación previo a la implantación del programa incluye la comparación de datos de } \\
\text { estadísticas nacionales y/o regionales con los datos a nivel del centro (ej. prevalencia de tabaquismo } \\
\text { en el centro comparado con nivel nacional) para definir aspectos a priorizar por constituir un área } \\
\text { especialmente deficitaria entre los y las trabajadoras del centro }\end{array}$ & 3 & 19 & $1^{\circ}$ & NP \\
\hline Hay interlocutores válidos para discutir los programas en el centro & 8 & 17 & $1^{\circ}$ & NP \\
\hline \multicolumn{5}{|c|}{ ADECUACIÓN } \\
\hline $\begin{array}{l}\text { Los componentes del programa se han definido de manera planificada, conforme a alguna estrategia } \\
\text { sistemática que ha orientado las decisiones para definir cada uno de los pasos a dar con el fin de } \\
\text { obtener los resultados buscados, y que no consisten en meras actuaciones aisladas o puntuales }\end{array}$ & 6 & 41 & $3^{\circ}$ & 6,7 \\
\hline $\begin{array}{l}\text { Los componentes del programa y las actividades a desarrollar se han planificado considerando su } \\
\text { adaptación a las personas a las que se dirigen (ej. con problemas crónicos de salud, disminución en } \\
\text { la agudeza visual, expuestas a determinadas condiciones de trabajo, a cargo de familiares depen- } \\
\text { dientes, entre otras) }\end{array}$ & 5 & 33 & $3^{\circ}$ & 8 \\
\hline $\begin{array}{l}\text { La intervención es multi-componente, incluye medidas sobre el ambiente laboral, la organización e } \\
\text { intervenciones individuales }\end{array}$ & 4 & 29 & $2^{o}$ & NP \\
\hline $\begin{array}{l}\text { 3. El plan de intervención ha sido revisado, pilotado y aprobado por un grupo de experto/as y } \\
\text { usuario/as }\end{array}$ & 3 & 25 & $2^{\circ}$ & NP \\
\hline
\end{tabular}


Tabla 3

Puntuaciones obtenidas por cada indicador de los $\mathbf{4}$ criterios priorizados (continuación)

\begin{tabular}{|l|l|l|l|l}
\hline & $\begin{array}{c}\text { Número de orden } \\
\text { en el que se expusieron }\end{array}$ & $\begin{array}{c}\text { Puntuación } \\
\text { media }\end{array}$ & $\begin{array}{c}\text { Tercil } \\
\text { para evaluar ese indicador }\end{array}$ & $\begin{array}{c}\text { Ítem de la lista de verificación } \\
\text { para }\end{array}$ \\
\hline ADECUACIÓN
\end{tabular}

7. La intervención se dirige no sólo al bienestar presente sino también a futuros riesgos y necesidades de los grupos de los y las trabajadoras

1. Hay una revisión sistemática de la evidencia científica sobre la efectividad de la intervención en EAS en el trabajo

2. La intervención utiliza un modelo conceptual de PSLT que orienta las decisiones sobre qué actuaciones hay que desarrollar (ej. modelo de teoría de autodeterminación)

El análisis de situación previo a la implantación del programa incluye la comparación de datos de estadísticas nacionales y/o regionales con los datos a nivel del centro (ej. prevalencia de tabaquismo en el centro comparado con nivel nacional) para definir aspectos a priorizar por constituir un área especialmente deficitaria entre los y las trabajadoras del centro

Hay interlocutores válidos para discutir los programas en el centro

\begin{tabular}{|l|l|l|l|l|}
\hline 7 & 24 & $1^{\circ}$ & NP \\
\hline 1 & 24 & $1^{\circ}$ & NP \\
\hline & 18 & $1^{\circ}$ & NP \\
\hline & 19 & $1^{\circ}$ & NP \\
\hline
\end{tabular}

9. Se adoptan métodos novedosos para la puesta en marcha de las intervenciones (ej. el modelo de intervención "hágalo usted mismo", identificando y capacitando recursos de la propia organización para el desarrollo de las intervenciones)

8. Las intervenciones parten de la identificación de "activos" y de "barreras" para el desarrollo del programa y usan estrategias para minimizar estas barreras

7. Las intervenciones incluyen acciones para conseguir la participación de los y las destinatarias y se les estimula para que piensen y transmitan nuevas y creativas soluciones para dar respuesta a sus necesidades

2. El programa utiliza las nuevas tecnologías (ej. redes sociales, internet, etc.) como herramientas innovadoras para ofrecer servicios

3. El proceso que se aplica para definir el programa y sus elementos es tan importante como el resultado final $\mathrm{y} / \mathrm{o}$ servicio que se ofrece

4. Se utiliza un modelo de activos en salud

12. En los programas de PSLT, las intervenciones incluyen la formación de profesionales sanitarios sobre prevención de fragilidad, multi-morbilidad, diagnóstico temprano y tratamiento del declive en la capacidad funcional, modelos de gestión de las condiciones crónicas de salud, etc.

10. Las intervenciones incluyen estrategias novedosas para mejorar la adherencia a los programas

5. Se identifican y evalúan indicadores de coordinación y encuentros de las personas que participan en la planificación del programa como número de reuniones, videoconferencias, fotos de equipo y otros

11. Se construyen Redes de conocimiento (de profesionales y de centros promotores del envejecimiento saludable) que trabajan en este ámbito, así como herramientas de difusión (web, rede sociales, etc.) de los detalles de las intervenciones que obtienen buenos resultados para prevenir el declive en la capacidad funcional asociado al envejecimiento

6. Se comunican las medidas en PSLT en el centro como "marca de empresa innovadora"

1. Existe un cambio en la política del centro ( no es esencial pero puede ser un indicador de innovación)

\begin{tabular}{|c|c|c|c|c|}
\hline & 9 & 49 & $3^{\circ}$ & 9 \\
\hline & 8 & 43 & $3^{\circ}$ & 11,12 \\
\hline & 7 & 41 & $3^{\circ}$ & 13,14 y 15 \\
\hline & 2 & 36 & $3^{\circ}$ & 9.c \\
\hline & 3 & 35 & $2^{\circ}$ & NP \\
\hline & 4 & 35 & $2^{\circ}$ & NP \\
\hline en & 12 & 32 & $2^{\circ}$ & NP \\
\hline & 10 & 28 & $2^{\circ}$ & NP \\
\hline$a$ & 5 & 27 & $1^{\circ}$ & NP \\
\hline & 11 & 27 & $1^{\circ}$ & NP \\
\hline & 6 & 20 & $1^{\circ}$ & NP \\
\hline ón & 1 & 3 & $1^{\circ}$ & NP \\
\hline
\end{tabular}




\begin{tabular}{|c|c|c|c|c|}
\hline \multicolumn{5}{|c|}{$\begin{array}{c}\text { Tabla } 3 \\
\text { Puntuaciones obtenidas por cada indicador de los } 4 \text { criterios priorizados (continuación) }\end{array}$} \\
\hline & $\begin{array}{l}\text { Número de orden } \\
\text { en el que se expusieron }\end{array}$ & $\begin{array}{l}\text { Puntuación } \\
\text { media }\end{array}$ & Tercil & $\begin{array}{l}\text { Ítem de la lista de verificación } \\
\text { para evaluar el indicador }\end{array}$ \\
\hline \multicolumn{5}{|c|}{ GARANTIA DE CALIDAD } \\
\hline $\begin{array}{l}\text { 10. Se mide y evalúa de forma sistemática el impacto que las intervenciones tienen en indicadores } \\
\text { claves de salud }\end{array}$ & 10 & 57 & $3^{\circ}$ & 16 \\
\hline $\begin{array}{l}\text { 1. Existe un sistema de recogida periódica de la información necesaria para evaluar el proceso } \\
\text { (sobre la forma en que se desarrollan las acciones para la promoción del envejecimiento saludable } \\
\text { en el trabajo) }\end{array}$ & 1 & 54 & $3^{\circ}$ & 17 \\
\hline $\begin{array}{l}\text { 5. Se han definido indicadores de proceso (ej. satisfacción con las actividades concretas, número de } \\
\text { personas que participan en el programa, número de medidas del entorno que se han adoptado, etc.) }\end{array}$ & 5 & 41 & $3^{\circ}$ & 18 \\
\hline $\begin{array}{l}\text { 10. Se mide y evalúa el impacto de la intervención en resultados organizacionales: tasa de absentis- } \\
\text { mo, rotación laboral, etc. }\end{array}$ & 10 & 36 & $3^{\circ}$ & 19 \\
\hline $\begin{array}{l}\text { 4. El programa de promoción del EAS incluye actuaciones en varios niveles: el entorno de trabajo, } \\
\text { la cultura corporativa, cambios organizacionales, e intervenciones dirigidas a nivel individual }\end{array}$ & 4 & 36 & $3^{\circ}$ & 10 \\
\hline $\begin{array}{l}\text { 3. Periódicamente el equipo del proyecto se reúne para evaluar la marcha del mismo y detectar si } \\
\text { existen elementos de mejora }\end{array}$ & 3 & 25 & $2^{\circ}$ & NP \\
\hline 6. Se evalúa el nivel de implantación de cada actividad planificada & 6 & 20 & $2^{\circ}$ & NP \\
\hline $\begin{array}{l}\text { 7. Se evalúa el nivel de satisfacción de los y las participantes con cada componente de la interven- } \\
\text { ción y su implicación/sensibilización con el programa }\end{array}$ & 7 & 20 & $2^{\circ}$ & NP \\
\hline $\begin{array}{l}\text { 11. El análisis de los resultados de la evaluación del programa se realiza diferenciando por grupos } \\
\text { como: sexo, niveles ocupacionales, etc. }\end{array}$ & 11 & 18 & $2^{\circ}$ & NP \\
\hline $\begin{array}{l}\text { 15. La evaluación de resultados incluye indicadores que permiten extraer conclusiones sobre el } \\
\text { impacto que el programa va a tener de cara a prolongar la edad laboral, y otros resultados especial- } \\
\text { mente relevantes para la población mayor trabajadora (ej. impacto en la prevención y/o gestión de } \\
\text { enfermedades crónicas en el ámbito laboral, etc.) }\end{array}$ & 15 & 14 & $1^{\circ}$ & NP \\
\hline $\begin{array}{l}\text { 2. La información se recoge en sistemas de registro adecuados para poder analizar cambios a lo } \\
\text { largo del tiempo }\end{array}$ & 2 & 14 & $1^{\circ}$ & NP \\
\hline 8.Se mide el grado de participación en diferentes fases y actividades del programa & 8 & 12 & $1^{\circ}$ & NP \\
\hline $\begin{array}{l}\text { 9. Se desarrolla una planificación temporal de las actividades, que permita identificar desviaciones } \\
\text { en las fechas propuestas para realizar cada medida }\end{array}$ & 9 & 5 & $1^{\circ}$ & NP \\
\hline 14. Se especifica la fecha exacta de cada actividad & 14 & 5 & $1^{\circ}$ & NP \\
\hline $\begin{array}{l}\text { 13. Se especifican puntos de corte en las medidas que se van a evaluar, por debajo de los cuales se } \\
\text { considera que no se obtienen los resultados esperados con el programa (ej. en qué porcentaje de } \\
\text { trabajadores/as tiene que observarse un aumento en los niveles de actividad física para considerar } \\
\text { que el programa ha tenido éxito) }\end{array}$ & 13 & 4 & $1^{\circ}$ & NP \\
\hline
\end{tabular}




\section{DISCUSIÓN}

Los resultados de nuestro estudio dan lugar a una matriz con los criterios relevancia, adecuación al objetivo, innovación y garantía de calidad y sus correspondientes indicadores así como a una herramienta en forma de lista de verificación que contribuyen a orientar las decisiones en planificación, diseño, implantación y evaluación de programas de PSLT dirigidos al EAS.

En un estudio con objetivos similares al nuestro se ofrece una guía para evaluar y desarrollar programas de PSLT, sin embargo se centra en un tipo de intervención muy concreta en este ámbito, consistente en incorporar incentivos económicos ${ }^{29}$. No tenemos conocimiento de que exista ningún otro estudio que evalúe globalmente todos los criterios de calidad propuestos en este trabajo, sino solamente estudios que valoran la efectividad de incorporar aspectos específicos que pueden facilitar la implantación de las medidas en los contextos laborales. Las investigaciones realizadas hasta la fecha se han centrado fundamentalmente en mostrar la relación entre efectividad de las intervenciones y el nivel de implicación de las personas destinatarias de los programas así como en analizar el apoyo y la relevancia otorgada por parte de las personas responsables de los centros como cuando, por ejemplo, se incorporan en las políticas de Seguridad y Salud en el Trabajo del centro o en otros protocolos de trabajo ${ }^{30-32}$. También existen estudios que muestran que cuando a las/os trabajadora/es se les pregunta, seleccionan objetivos de intervención adecuados ${ }^{33}$. Estos aspectos constituyen estrategias incluidas en el criterio de relevancia identificado en nuestro estudio.

Por otra parte, son numerosos los estudios que analizan la utilidad de implementar intervenciones multi-componentes ${ }^{34}$ y centradas, no sólo en conseguir cambios comportamentales individuales, sino también cambios del ambiente físico y psicosocial del trabajo ${ }^{32,35}$. Otros contemplan la utilidad de incluir en los programas sistemas de evaluación que per- mitan extraer conclusiones sobre el proceso de implantación y no sólo de los resultados ${ }^{36}$. Por último, algunos estudios sobre programas de PSLT por una parte señalan la necesidad de identificar elementos facilitadores así como barreras, con el fin de que guíen la implementación de los programas y, por otra, la de explorar la relación entre efectividad de los programas y la metodología seguida para su implementación.

A la luz de estos estudios que se centran en la valoración de aspectos concretos de las intervenciones, se plantea la utilidad de contar con una opinión consensuada acerca de los aspectos claves que resultan importantes a considerar en los programas, así como de un esquema que ayude a sistematizar la metodología global de implantación de las intervenciones. Este esquema debería incluir los elementos que se han identificado como factores de éxito de las intervenciones, facilitando de esta manera las evaluaciones de proceso y no sólo de resultados de los programas ${ }^{36}$.

Los resultados de nuestro estudio pueden ser útiles en este sentido al proponer una matriz de criterios e indicadores y una lista de verificación, las cuales contribuyen a orientar la toma de decisiones en los programas de PSLT dirigidos al EAS. Estas herramientas, generadas mediante un proceso sistemático de recogida de opinión y valoración de expertos/as, facilitarán a las personas interesadas la puesta en marcha de actuaciones de PSLT guiándolas en la toma de decisiones. Por otra parte, este estudio se realizó en el conjunto de países seleccionados, por lo que sus resultados podrían no ser directamente aplicables en otros. Por ello, sería necesario contar con futuras investigaciones que pongan en práctica los instrumentos generados en este estudio Delphi para planificar y evaluar programas concretos de promoción de salud en los lugares de trabajo dirigidos al envejecimiento activo y saludable, con objeto de depurarlos y valorar la implementabilidad y utilidad de los diferentes criterios en contextos laborales concretos. 


\section{AGRADECIMIENTOS}

A Julia Bolívar Muñoz, por su participación en el análisis de los datos obtenidos en el Delphi y la elaboración del informe final.

A las personas responsables de los equipos del proyecto PROGRESS: Elspeth Gibson, Rosa Juarez, Luise Ebenbeck, Anton Petrenko y Mauro Calise, por su ayuda en la organización del trabajo de campo del estudio en los distintos países.

\section{BIBLIOGRAFÍA}

1. Organización Mundial de la Salud. Active Ageing: A policy Framework. Ginebra:OMS;2012.

2. Eurostat. Statistics explained. Population structure and ageing. Disponible en: http://ec.europa.eu/eurostat/ statistics-explained/index.php/Population_structure_and_ ageing.

3. Instituto Nacional de Estadística (INE). Proyección de la Población Española a corto plazo 2013-2023. Madrid: INE; 2013. Disponible en: www.ine.es

4. Comisión Europea. European Year for Active Ageing and Solidarity between Generations, 2012. Bruselas: Comisión Europea; 2012. Disponible en: http://www.age-platform. eu/images/stories/EN/ey2012_joint_leaflet-en.pdf

5. Siegrist J. Quality of work, health and early retirement: European comparisons. En: A. Börsch-Supan et al (eds.), The individual and the Welfare State. Life Histories in Europe. Heidelberg: Springer-Verlag; 2011.

6. Comisión Europea. The 2015 ageing report. Underlying assumptions and projection methodology. European Economy $8 / 2014$.

7. Boletín Oficial del Estado. Ley 27/2011 sobre adecuación, adaptación y modernización del Sistema de Seguridad Social. BOE núm 184 de 2/8/2011.

8. Boletín Oficial del Estado. Estrategia Global para el Empleo de los Trabajadores y las Trabajadoras de Más Edad 2012-14. BOE núm 283, de 24/11/2011.

9. Marmot M. Fair Society, Healthy Lives: The Marmot Review. Strategic Review of Health Inequalitites in England post-2010. London: Institute of health equity; 2010.

10. Instituto Nacional de Estadística (INE). Mujeres y hombre en España. Salud (actualizado a mayo de 2015). Esperanza de vida en buena salud. Madrid: INE; 2015. Disponible en: www.ine.es
11. Barnay T. Effects of health on the labour force participation of older persons in Europe. Question d'economie de la santé. 2006.109.

12. Ministerio de Sanidad, Servicios Sociales e Igualdad. Encuesta Nacional de Salud de España 2011/12. Madrid: Ministerio de Sanidad, Servicios Sociales e Igualdad e Instituto Nacional de Estadística;2012.

13. Morschhäuser M. Healthy work in an aging Europe. Essen: Federal Association of Company Insurance Funds; 2006.

14. Mateo I. Experiencias y estrategias para la gestión de la edad en la empresa. En: Informe Sobre el estado de la seguridad y salud laboral en España 2010, Madrid: INHST; 2012.

15. Anderson J, Parker W, Steyn N, Grimsrud A et al. Interventions on diet and physical activity: What works. Ginebra: Organización Mundial de la Salud; 2011.

16. Tremblay PA, Nobrega S, Davis L, Erck E, Punnett L. Healthy workplaces? A survey of Massachusetts employers. Am J Health Promot. 2013 Jul-Aug; 27(6): 390-400.

17. Tuah NAA, Amiel C, Qureshi S, Car J, Kaur B, Majeed A. Transtheoretical model for dietary and physical exercise modification in weight loss management for overweight and obese adults (Review). Cochrane Database of Systematics Review. 2011; (10). JohnWiley \& Sons, Ltd; 2011.

18. Fortier M, Duda JL, Guerin E and Teixeira P. Promoting physical activity: development and testing of selfdetermination theory-based interventions (Review). Int $\mathrm{J}$ of Behav Nutr and Phys Act. 2012; 2: 9-20.

19. Progress Project. Disponible en: http://www.progresshealthyageing.eu/

20. Rym B, Hendy A, Marine L,Olivier S, Corinne A. Using and Reporting the Delphi Method for Selecting Healthcare Quality Indicators: A Systematic Review. Plos One, June2011,(6):6:e20476.

21. Hsu C-C, Sandford BA. The Delphi technique: making sense of consensus. Pract Assess Res Eval. 2007; 12 (10).

22. Daponte A, Bernal M, Bolívar J, Mateo I et al. "Criteria for implementing interventions to reduce health inequalities in primary care settings in European regions". Eur J Public Health. 2014 Dec 16;24(6):980-90

23. Centers for Disease Control and Prevention (CDC). A framework for program evaluation in public health. Washington, DC: Centers for Disease Control and Prevention; 1999. Disponible en: http:/www.cdc.gov/ mmwr/preview/mmwrhtml/ rr4811a1.htm\#box13. 
24. Rychetnik L, Frommer M, Hawe P, Shiell A. Criteria for evaluating evidence on public health interventions. J Epidemiol Community Health. 2002; 56:119-27.

25. Craig P, Dieppe P, Macintyre S, Michie S, Nazareth I, Petticrew M. Medical Research Council Guidance. Developing and evaluating complex interventions: the new Medical Research Council guidance. BMJ. 2008; 29:337:a1655. doi: 10.1136/bmj.a1655.

26. May AK, Cuschieri J, Johnson JL, Duane TM, Cherry-Bukowiec JR, Rosengart MR. Determining a core curriculum in surgical infections for fellowship training in acute care surgery using the Delphi technique. Surg Infect (Larchmt). 2013; 14(6):547-53.

27. Cheng EM, Crandall CJ, Bever CT Jr, Giesser B, Haselkorn JK, Hays RD, et al. Quality indicators for multiple sclerosis. Mult Scler. 2010 Aug; 16(8):97080 .

28. Rosengart MR, Nathens AB, Schiff MA. The identification of criteria to evaluate prehospital trauma care using the Delphi technique. J Trauma. 2007 Mar; 62(3):708-13.

29. Consensus Statement of the Health Enhancement Research Organization; American College of Occupational and Environmental Medicine; American Cancer Society and American Cancer Society Cancer Action Network. Guidance for a reasonably designed, employer-sponsored wellness program using outcomes-based incentives. J Occup Environ Med. 2012; 54(7):889-96.

30. Brace AM, Padilla HM, DeJoy DM, Wilson MG, Vandenberg RJ, Davis M. Applying RE-AIM to the Evaluation of FUEL Your Life: A Worksite Translation of DPP. Health Promot Pract 2015; 16 (11): 28-35.

31. Cancelliere C, Cassidy JD, Ammendolia C, Côté P. Are workplace health promotion programs effective at improving presenteeism in workers? a systematic review and best evidence synthesis of the literature. BMC Public Health. 2011, 11:395.

32. Blackford K, Jancey J, Howat P, Ledger M, Lee AH. Office-based physical activity and nutrition intervention: barriers, enablers, and preferred strategies for workplace obesity prevention, Perth, Western Australia, 2012. Prev Chronic Dis 2013 Sep 12; 10: E154.

33. Kilpatrick M, Sanderson K, Blizzard L, Nelson M, Frendin S, Teale B, et al. Workplace health promotion: what public-sector employees want, need, and are ready to change. J Occup Environ Med. 2014; 56(6) : 645-51.

34. Ausburn TF, LaCoursiere S, Crouter SE, McKay T. Review of worksite weight management programs. Workplace Health Saf. 2014 Mar; 62(3): 122-6.
35. Nelson CC, Wagner GR, Caban-Martinez AJ, Buxton OM, Kenwood CT, Sabbath EL, et al. Physical activity and body mass index: the contribution of age and workplace characteristics. Am J Prev Med. 2014 Mar; 46(3 Suppl 1): S42-51.

36. Wierenga D, Engbers LH, Van Empelen P. What is actually measured in process evaluations for worksite health promotion programs: a systematic review. BMC Public Health. 2013 Dec 17; 13:1190. 


\section{Anexo 1 \\ Lista de verificación para el diseño y evaluación de programas para el envejecimiento saludable en el trabajo}

Esta lista de verificación incluye preguntas sobre elementos claves para evaluar los programas para favorecer el envejecimiento activo y saludable (EAS) en el trabajo. En el caso de que las intervenciones que se estén desarrollando no cumplan con alguna de los ítems que se evalúan se proponen una serie de recomendaciones.

1- ¿Existe en el Centro de trabajo un documento donde se recoge de manera explícita el compromiso por desarrollar acciones dirigidas a sensibilizar y apoyar a las personas empleadas mayores de 45 años a adoptar conductas saludables? (Criterio RELEVANCIA. Indicador: 1).

a- Sí. (Aportar evidencia).

b- No. Recomendación: Identificar documentación corporativa donde incorporar dicho compromiso: Política explicita de EAS, Política de Seguridad y Salud en el Trabajo, Convenio colectivo u otros

2- ¿La dirección del centro destina recursos humanos y materiales específicos y suficientes para desarrollar actuaciones de EAS? (Criterio RELEVANCIA. Indicador 1).

a- Si. (Aportar evidencia).

b- No. Recomendación: Identificar recursos personales y materiales del centro para el desarrollo de programas de EAS

3- ¿Para la definición del programa se ha incorporado información sobre posibles necesidades actuales y futuras relacionadas con la estructura de la edad? ej. Proyecciones de edad de la plantilla del centro, sobre indicadores de absentismo o de salud (Criterio RELEVANCIA. Indicador 2).

a- Si. (Aportar evidencia).

b- No. Recomendación: Identificar fuentes de información disponibles: desde personal, servicio de vigilancia de la salud y otras; o bien la necesidad de incluir nuevos sistemas de registro.

4-. Las personas del centro a las que se dirige de manera prioritaria el programa se han identificado y priorizado porque cumple con características que les hace especialmente vulnerable. Ej. Ocupaciones de mayor riesgo, trabajadore/as más mayores, presencia de enfermedad crónica o discapacidad. (Criterio RELEVANCIA. Indicador 9).

a- Si. (Aportar evidencia).

b- No. Recomendación: Identificar grupos de especial vulnerabilidad, aquellos con mayor prevalencia de bajas por enfermedad, los más mayores, personas con discapacidad, sectores con mayor accidentabilidad, y otros

5- ¿Para decidir las acciones concretas que se van a desarrollar para promover el EAS en el trabajo se han realizado algunas de las siguientes acciones? (Las respuestas que siguen no son excluyentes, puede contestar todas las que procedan) (Criterio RELEVANCIA indicadores 2, 5, 9).

5. a- Un análisis de datos de salud de los y las trabajadoras.

a. Si. (Aportar evidencia).

b. No. Recomendaciones: Realizar un análisis de Vigilancia de la Salud colectiva de los y las trabajadoras.

5. b- Un análisis de datos de la organización que indican áreas de mejora o de especial dificultad.

a. Si. (Aportar evidencia).

b. No. Recomendaciones: Solicitar al Departamento de personal información sobre tasas de absentismo, rotación de puestos, y otras que indique áreas del centro más problemáticas.

5. c- Recogida de propuesta de los y las trabajadoras del centro, a partir del análisis de sus preferencias

a. Si. (Aportar evidencia).

b. No. Recomendaciones: Realizar una exploración de propuestas de trabajadores y trabajadoras a través de encuestas, entrevistas grupales, u otras. 
5. d- A propuesta de profesionales de Salud laboral, u otros grupos de interés del centro.

a. Si. (Aportar evidencia).

b. No. Recomendaciones: Realizar una exploración de grupos de interés a través de encuestas, dinámicas de grupo estructuradas, u otras.

5. e- Análisis de las características de la plantilla, identificando los grupos de especial vulnerabilidad en el centro que deben ser priorizados en el desarrollo de intervenciones.

a. Si. (Aportar evidencia).

b. No. Recomendaciones: Realizar una exploración del ajuste puesto/persona: de personas de más edad en puestos con mayores exigencias físicas y/o mentales, en situación de diversidad funcional, población inmigrante, y otras.

$$
\text { 5. f- Otras }
$$

6- Existe una planificación de las actuaciones en Promoción del envejecimiento saludable por escrito que incluye los objetivos/resultados a obtener, las medidas/acciones concretas a adoptar, las personas responsables de las mismas y la temporalización de estas medidas (Criterio ADECUACION. Indicador 6 )

a Sí. (Aportar evidencia).

b. No. Recomendación: Planifique el programa a implantar definiendo los objetivos, personas a las que se dirige, las medidas y acciones concretas, las personas responsables de cada acción, el cronograma de trabajo y los indicadores de evaluación de proceso y de resultado. : (Las respuestas que siguen no son excluyentes, puede contestar toda

7- ¿El diseño del programa utiliza un modelo teórico/conceptual con suficiente evidencia científica sobre la eficacia de las actuaciones propuestas? (Criterio ADECUACION. Indicador 6).

a- Sí. . (Aportar evidencia).

b.No. Recomendación: Utilice un modelo conceptual con soporte científico para obtener el cambio conductual que se pretende.

8- ¿A la hora de definir los objetivos y componentes del programa se han considerado segmentos de población diferentes dentro del centro? ej. Grupos con enfermedad crónica versus sin enfermedad crónica, hombres y mujeres, grupos de clases ocupacionales, etc. (Criterio ADECUACION Indicador 5).

a- $\mathrm{Si}$, se han identificado segmentos de población diferentes a los que se dirige componentes específicos del programa. (Aportar evidencia).

b- No, la intervención es la misma para todo/as lo/as trabajadore/as del centro. Recomendación: analizar los resultados del programa de manera desagregada con objeto de identificar si su impacto es diferente en algunos grupos de trabajo y planificar acciones específicas.

9- El programa incluye componentes innovadores como: (Criterio INNOVACIÓN. Indicadores 9, 1).

9. a- Identificación conjunta con agentes claves del centro de los aspectos del entorno y puesto de trabajo que deben ser modificados para permitir que los y las trabajadoras más mayores se mantengan activos y saludables en su trabajo durante más tiempo.

a. Si. (Aportar evidencia).

b. No .Recomendación: Elaborar un mapa de activos para la salud en el centro.

9. b. Medidas sobre la organización y relaciones sociales en el trabajo que constituyan oportunidades para una mejor gestión del trabajo por parte de trabajadores/as más mayores

a. Si. (Aportar evidencia).

b. No. Recomendaciones: Identificar medidas organizacionales y sociales (ej. Flexibilidad en horario, equipos de trabajo, club social para alimentación saludable o prácticas deportivas).

9. c- Uso de nuevas tecnologías, redes sociales, etc.

a. Si. (Aportar evidencia).

b. No. Recomendación: Identificar aplicaciones tecnológicas de uso más habitual entre trabajadore/as y explorar su aplicación con el objetivo del programa. 
9. e- Uso del modelo de intervención en PST del "hágalo usted mismo" mediante la capacitación de personas en la empresa sobre envejecimiento saludable (ej. profesionales de vigilancia de la salud)

a. Si. (Aportar evidencia).

b. No. Recomendación: Identificar a personas de la organización que por su perfil profesional y motivacional puedan ser formadas en EAS para la implantación de los programas.

9. f- Se implantan otras medidas novedosas. ¿Cuales?

10- ¿Cuáles de los siguientes componentes incluye la intervención? (Criterio GARANTIA CALIDAD. Indicador 4). 10. a- Medidas sobre el entorno y puestos de trabajo.

a. Si. (Aportar evidencia).

b. No .Recomendaciones: Incorporar acciones sobre los lugares de trabajo (ej. Disponer de cartelería con mensajes saludables, vigilar los productos de las máquinas expendedoras, y otras).

10. b- Medidas sobre la organización del trabajo y relaciones sociales en el trabajo

a. Si. (Aportar evidencia).

b. No. Recomendaciones: Definir estrategias que den oportunidad para adoptar hábitos saludables como distribución de los horarios de trabajo de forma que sean compatibles con patrones de alimentación adecuados (acudir al propio domicilio).

10. c- Medidas para apoyar cambios en los estilos de vida mediante estrategias de sensibilización, adquisición de habilidades, superación de barreras individuales, etc. ¿cuales?

a. Si. (Aportar evidencia).

b. No. Recomendaciones: Realizar talleres formativos, medidas sobre consejo breve en salud, y otras acciones de formación.

11- Existe un análisis de los elementos del entorno que favorecen o dificultan la adopción de hábitos saludables. (Criterio INNOVACION. Indicador 8).

a. Si. (Aportar evidencia).

b. No. Recomendación: Aplicar una lista de verificación de oportunidades del entorno para la facilitar la adopción de conductas saludables e identficarlos.

12- ¿Se han identificado barreras y activos para el desarrollo de los programas? Ej. Falta de motivación para participar en los programas, dificultades para la incorporación a componentes concretos por ejemplo por analfabetismo digital en algunos grupos, falta de tiempo, etc. (Criterio INNOVACION Indicador 8).

a-Sí. (Aportar evidencia).

b.No. Recomendación: realizar un estudio para identificar barreras y dificultades para implantar acciones y para la adherencia a los componentes del programa.

13- ¿Se ofrece a las personas participantes la posibilidad de expresar sus opiniones y desarrollar su potencial? ¿Se da la oportunidad a las personas a contribuir al programa con sus propias ideas y recursos? ¿o a jugar un papel significativo en la planificación del programa 0 a incorporar soluciones que se desarrollan como parte del programa? (Criterio INNOVACION Indicador 7).

a- Si. (Aportar evidencia).

b- No. Recomendación: Incorporar medios para conocer la opinión de los y las trabajadoras sobre el programa en las fases iniciales del mismo: buzón de sugerencias, entrevistas personales, y otras.

14- ¿Se ha difundido dentro de la Organización los programas y estrategias que se están desarrollando? INNOVACION. Indicador 7.

a- Sí. . (Aportar evidencia).

b.No. Recomendaciones: Antes de comenzar, difundir el programa usando los canales habituales de comunicación del centro.

15- ¿Existe alguna estrategia o medida para que las personas empleadas puedan hacer sugerencias o expresar su opinión sobre los programas? (Criterio INNOVACION Indicador 7).

a. Si. (Aportar evidencia).

b- No. Recomendación: Generar canales de comunicación hacia arriba abiertos de manera permanente. 
16- ¿Existe una evaluación del impacto de las intervenciones sobre indicadores de salud, que se definieron como objetivos del programa? (ej. Salud percibida, cambios en hábitos, ICL...) (Criterio GARANTIA CALIDAD. Indicador 10).

a- Sí. . (Aportar evidencia).

b- No. Recomendación: Realizar una evaluación pre y post intervención incluyendo variables de salud como: salud percibida, resultados de exámenes de salud, prevalencia de hábitos saludables y otras medidas.

17- ¿Se han identificado indicadores de proceso?

a- Sí. (Aportar evidencia).

b- No. Recomendación: Realizar una evaluación de la forma en que se desarrolla el programa incluyendo: número de participantes en las distintas actividades programadas, satisfacción con las acciones realizadas, y otros indicadores de proceso.

18- ¿Se han definido canales para la evaluación de proceso? (ej. Reuniones de seguimiento del grupo planificador, participación en las sesiones, cuestionarios de satisfacción de trabajadores/as con las medidas adoptadas,

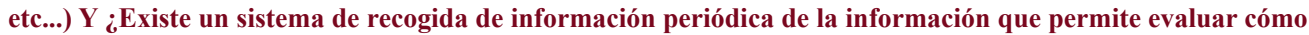
se van desarrollando el programa, con objeto de identificar desviaciones respecto a lo planificado y adoptar medidas para su corrección? Criterio GARANTIA DE CALIDAD. Indicador 1)

a- Sí. (Aportar evidencia).

b- Se han definido los sistemas de recogida de información pero no se ha realizado la evaluación de proceso. Recomendación: analizar la información disponible.

c. No existen los canales ni la evaluación. Recomendación: Incorporar sistemas de recogida de información sobre el programa y analizar la información de manera periódica.

19- ¿Existe una evaluación del impacto del programa en indicadores organizacionales?

a- Sí. (Aportar evidencia).

b- No. Recomendación: Evaluar el impacto del programa en indicadores de la organización como: tasa de absentismo laboral en el último año, nivel de satisfacción laboral, rotación de trabajadores, quejas y reclamaciones, indicadores de productividad, y otros. 